\title{
Immune System dysfunction in patients with Infective Endocarditis
}

\author{
Safa Meshaal ${ }^{1}$, Marwa Meshaal ${ }^{1}$, and Rabab El Hawary ${ }^{2}$ \\ ${ }^{1}$ Cairo University Kasr Alainy Faculty of Medicine \\ ${ }^{2}$ Cairo University
}

May 5, 2020

\begin{abstract}
Background: Infective endocarditis (IE) is a severe disease with high morbidity and mortality rate. Poor immune response and autoimmunity have been reported in patients with IE and result in different complications such as severe sepsis, glomerulonephritis, arthritis, coagulopathy and embolization. This study aims to investigate the role of immune system in the pathogenesis of IE through assessment of memory and naïve $\mathrm{T}$ and $\mathrm{B}$ lymphocytes and T regulatory cells ( $\mathrm{T}$ regs). Methods: Twenty IE patients and twenty healthy age and sex matched donors were included in the study. Flow cytometry was used to asses the naïve and memory subsets of peripheral blood lymphocytes using cell surface markers CD4, CD19, CD45RO, CD45RA, CD27 and IgD. $\mathrm{T}$ regs were characterized by co-expression of CD25/FoxP3 among the CD4+ T cells. Results: IE patients had significant lower number of CD45RO+CD4+ memory $\mathrm{T}$ cells when compared to healthy control $(\mathrm{p}=0.03)$. No differences were observed regarding CD45RA+CD4+ naïve T cells, CD19+CD27+ memory B cells and CD19+IgD+ naïve B cells between the IE patients and healthy control. However, IE patients had significantly lower percentage and number of $\mathrm{T}$ regs $(\mathrm{p}=0.039$, $\mathrm{p}=0.009$ respectively). Conclusion: IE patients suffer from immune system dysfunction which contribute to the disease course and complications. The decrease in the CD45RO+CD4+ memory $\mathrm{T}$ cells can partly explain why IE patients cannot effectively clear the infection before the invading pathogen replicate and cause tissue damage. The defect in $\mathrm{T}$ regs may underlie the autoimmunity and occurrence of autoantibodies reported in IE patients
\end{abstract}

\section{Introduction:}

Infective endocarditis (IE) is a life-threatening multisystem disease with high morbidity and mortality. Patients who survive, usually suffer from log-lasting effects in spite of the advances in diagnostic imaging, improved antimicrobial therapy, and potentially curative surgery (1-4).

Crude incidence of the global burden of IE was estimated to range from 1.5 to 11.6 cases per 100,000 personyear (5). Since first described by Osler in nineteenth century, the natural history of the disease, its sequalae and risk factors have remarkably changed $(6,7)$.

Patients with IE suffer from various manifestations which reflect immune system abnormalities including arthritis, vasculitis, immune-complex mediated glomerulonephritis, embolism and dermatomyositis-like syndrome (8-10).

Furthermore, various autoantibodies have been frequently detected in patients with IE including antinuclear antibodies, anti-cardiolipin IgG and IgM, anti-RO and anti-La, anti-neutrophil cytoplasmic antibodies, and anti-cyclic citrullinated peptides $(8,11)$. Higher serum concentrations of IL- $1 \beta$, TNF- $\alpha$ and IL-12 were also reported in patients with IE when compared to healthy controls (12). In addition, T regulatory cells (CD4+CD25+FoxP3+ cells) are increased in patients with Q fever endocarditis (13). Although immune system (IS) implication in pathogenesis of IE is very likely, studies investigating its role were very limited. 
In the present study, we investigated different $\mathrm{T}$ and $\mathrm{B}$ lymphocytes subsets including memory, naïve and regulatory $\mathrm{T}$ cells and their possible role in pathogenesis of IE.

2. Patients and Methods:

\subsection{Study groups:}

Twenty patients (11 males, 9 females) diagnosed with definite IE who were referred to the Kasr Al-Ainy IE Working Group in the period from 2018 through 2019 were included in this study. Diagnosis was based on American College of Cardiology/American Heart Association (AHA/ACC) guidelines and European Society of Cardiology (ESC) guidelines for the diagnosis and management of IE patients $(14,15)$. Their age ranged between 14-58 years. Twenty-two healthy age and sex matched volunteers served as control group. Patients were subjected to thorough history taking and meticulous examination. All samples were withdrawn after patients' consents were obtained. The Study was approved by Kasr Al-Ainy Ethical Committee.

\subsection{Flow cytometry (FCM) for Lymphocyte subsets enumeration :}

Peripheral blood in EDTA was collected from patients and controls and analysis of lymphocytes was done within 2 hours from collection using surface-stain with PE.Cy5.1-conjugated anti-CD19 (J3-119), PECy7-conjugated CD4. For evaluation of naïve and memory T cells, we used FITC-conjugated CD45RA, PEconjugated CD45RO respectively, whereas naïve and memory B cells were evaluated by FITC-conjugated IgD and PE-Cy7-conjugated CD27 respectively. Alexa Fluor@ 647 -conjugated anti-Foxp3 antibodies and PE-conjugated CD25 were used to assess the T regulatory cells (Tregs) following permeablization and fixation using IntraPrep permeablization Reagent (Beckman Coulter IM2389). All monoclonal antibodies were purchased from Beckman Coulter (Kendall, FL) except IgD (Dako cat. F0189) and Alexa Fluor@ 647 mouse anti-Human Foxp3 (BD Biosciences Cat No.561184). Approximately 20,000 labeled cells were analyzed through a CYTOMICS FC 500 Flow Cytometer (Beckman Coulter Kendall, FL) and CXP Software version 2.2 .

\subsection{Statistical Analysis:}

Data were statistically described in terms of mean \pm standard deviation $( \pm \mathrm{SD})$, median and range, or frequencies (number of cases) and percentages when appropriate. Comparison of numerical variables between the study groups was done using Student $t$ test for independent samples in comparing 2 groups when data was parametric and using Mann Whitney test when data was nonparametric. p values less than 0.05 were considered statistically significant. Correlations were calculated using Point-Biserial correlation. All statistical calculations were done using computer program SPSS (Statistical Package for the Social Science; SPSS Inc., Chicago, IL, USA) release 15 for Microsoft Windows (2006).

\section{Results:}

3.1. IE patients' characteristics, clinical and basic laboratory features:

Twenty IE patients were included in the study; 11 males and 9 females. Their age ranged between 16-58 years (mean 29.1 12.5 years). IE on native valve was documented in $85 \%(17 / 20)$ of patients while five patients $(25 \%)$ had IE on prosthetic valve. Only one patient had previous history of IE. Fifteen patients $(75 \%)$ had large vegetations ( $>1 \mathrm{~cm}$ on mitral/aortic valve or $>2 \mathrm{~cm}$ on tricuspid valve) and eleven patients $(55 \%)$ suffered from multiple vegetations. Splenomegaly was present in seventeen patients (75\%). Fourteen (70\%) patients suffered from embolization and eight patients (40\%) had renal impairment. The disease course was complicated by severe sepsis in three patients (15\%) (Fig 1). Surgical intervention was needed in 14 patients (70\%) while mortality rate reached $35 \%$.

Blood culture revealed no growth in seven patients (35\%), methicillin-resistant Staphylococcus aureus (MRSA) in four patients (25\%), Oxacillin-sensitive Staphylococcus aureus (OSSA) in four patients (25\%), aspergillus in two patients (10\%), Klebsiella in one patient (5\%), Bartonella in one patient (5\%) and Streptococcus pneumoniae in one patient (5\%) (Fig 2). 
Anemia was present in all patients, total leucocytic count (TLC) was elevated (mainly polymorphonuclear cells) in twelve patients $(60 \%)$. TLC showed a mean of $12.8 \times 10^{3} \pm 7.4 \times 10^{3}$ in IE patients and $7.8 \times 10^{3} \pm 5 \times 10^{3}$ in healthy controls. TLC was statistically higher in IE patients when compared to healthy control $(\mathrm{p}=0.028)$.

Thrombocytopenia was observed in four patients (20\%). C-reactive protein on admission was constantly elevated (mean $107 \pm 58.78 \mathrm{mg} / \mathrm{dl}$ ).

\section{2. $\mathrm{T}$ helper maturation :}

Percentage of CD4 helper cells was $39.8 \pm 9.9 \%$ in IE patients and $40.8 \pm 8.3 \%$ in healthy control with no significant difference between the 2 groups $(\mathrm{p}=0.7)$. Similarly, the absolute count of CD4 helper T cells was very close in IE patients and the control group (927 $\pm 511,964 \pm 287$ respectively, $\mathrm{p}=0.7)$.

Expression of CD45RA and CD45RO was assessed on CD4+ cells as markers for naïve and memory subsets respectively. Percentage of $\mathrm{CD} 4+45 \mathrm{RO}+$ memory $\mathrm{T}$ cells showed a mean value of $48.3 \pm 21 \%$ in IE patients and $58.63 \pm 16.26 \%$ in healthy controls. Comparing the patients versus the controls revealed no significant statistical difference $(\mathrm{p}=0.08)$. Absolute count of $\mathrm{CD} 4+\mathrm{CD} 45 \mathrm{RO}+$ was significantly lower in the IE patients when compared to control $(391 \pm 235.9,559 \pm 240.6$ respectively) with $\mathrm{p}=0.03$ (Fig 3).

Negative correlations between absolute number of $\mathrm{CD} 4+45 \mathrm{RO}+$ memory $\mathrm{T}$ cells and disease severity indicators (severe sepsis, death and unfavorable outcome) were detected. However, none of the correlation was significant $(\mathrm{r}=-0.41,-0.2,-0.3$ respectively).

Percentage of CD4+45RA+ cells showed a mean value of $40.2 \pm 18.7 \%$ in IE patients and $35.67 \pm 15.85 \%$ in healthy controls. Comparing the patients versus the controls revealed no significant statistical difference $(\mathrm{p}=0.4)$. Absolute count of CD4+CD45RA+ was similar in both groups, $400 \pm 285.9$ the IE patients and $336 \pm 197.4$ in control group with $\mathrm{p}=0.4$.

\subsection{T regulatory cells $(\mathrm{CD} 4+\mathrm{CD} 25+\mathrm{FoxP} 3)$ :}

Percentage of CD4+CD25+FoxP3 cells showed a mean value of $1.4 \pm 1.06 \%$ (median $1.2 \%$ ) in IE patients and $2.2 \pm 0.96 \%$ (median $2.3 \%$ ) in healthy controls. T regs percent was significantly lower in IE patients $(\mathrm{p}=0.039)$.

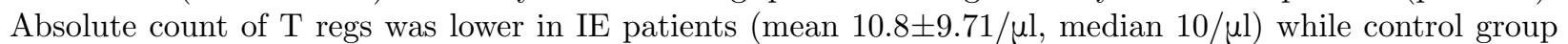
showed mean of $(20.7 \pm 10.6 / \mu l$, median $20 / \mu l)$ with statistically significant difference $(p=0.009)$ (Fig 3$)$.

\subsection{B lymphocytes maturation:}

Percentage of CD19+ B cells were very close in both groups; showed a mean value of $9.9 \pm 5.5 \%$ in IE patients and $10.5 \pm 4.3 \%$ in healthy controls; $(\mathrm{p}=0.7)$.

Absolute CD19 count showed a mean value of $241 \pm 171 / \mu$ in IE patients and $256 \pm 167 / \mu$ in healthy controls with $(\mathrm{p}=0.8)$.

B cell maturation was assessed for IE patients and control group. Percentage of IgD+ CD27- cells (naïve B cells) showed a mean value of $16.2 \pm 21 \%$ in IE patients and $19.1 \pm 19.3 \%$ in healthy control. Comparing the patients versus the controls, no significant statistical difference was obtained $(\mathrm{p}=0.6)$. Absolute count of IgD+ B cells was also close in both groups; $44 \pm 67.5 / \mu \mathrm{l}$ in IE patients and $45.6 \pm 50.6 / \mu$ l in healthy control $(\mathrm{p}=0.9)$

Memory B cells (CD27+IgD-) were similar in both groups in terms of percentage as well as absolute count. Percentage of memory B cells showed a mean value of $37.4 \pm 18.5 \%$ in IE patients and $16.75 \pm 13.13 \%$ in healthy controls; $(\mathrm{p}=0.4)$. Absolute count of memory B cells was $100.4 \pm 99 / \mu \mathrm{l}$ in IE patients and $88 \pm 57.5 / \mu \mathrm{l}$ in healthy control $(\mathrm{p}=0.6)$.

4. Discussion:

In spite of the advances in therapeutic approaches and early diagnosis, IE is still a challenging disease with multisystem affection and complications (3). The clinical course of IE patients largely depends on the inflammatory response and immune system reaction to the invading organism with variable degrees of 
hemodynamic and metabolic compromise (16). Several studies referred to the implication of immune system dysfunction in different morbidities and complications of IE $(8,10,12,17)$. Immune complexes formation and occurrence of auto-antibodies of low specificity were reported in IE patients repeatedly $(8,9,18)$. In the present study, we have investigated $\mathrm{T}$ and $\mathrm{B}$ lymphocytes subsets including naïve, memory and regulatory $\mathrm{T}$ cells in patients with IE and matched healthy control.

CD4+ CD45RO + memory T cells were significantly lower in terms of absolute count in IE patients compared to the control $(\mathrm{p}=0.003)$, however there was no difference regarding naïve $\mathrm{CD} 4 \mathrm{~T}$ cells between the patients and control. Upon exposure to antigen during an infection, antigen specific T cells are activated, proliferate and differentiate into effector cells which act to clear the infection and subsequently, most of these cells die. The surviving cells are memory cells which can provide an enhanced protective response upon re-exposure to the same pathogen.

In normal adults, the CD4 memory $\mathrm{T}$ cells repertoire is higher than the original naïve $\mathrm{T}$ cells. This higher frequency of antigen specific cells increases the likelihood that any re-infection will be detected quickly and more efficiently, allowing the immune response to get underway before the pathogen has time to replicate extensively or cause substantial damage (19). These activated cells can then either attack the invading organism directly or provide help to B cells or cytotoxic T cells (20). Studies reported that memory CD4 T cells defect in old age resulted in decrease in antigen-specific B cell expansion and decrease IgG production (21). The defect in CD4 memory cells in IE patients reported in this study may reflect a defect in the immune response to the invading pathogen explaining why those patients can not eliminate the infection properly and hence pass through a severe course with high complication rate.

We also reported a defect in $\mathrm{T}$ regs $(\mathrm{CD} 4+\mathrm{CD} 25+\mathrm{FoxP} 3+$ cells $)$ in IE patients in terms of percentage and absolute count. $\mathrm{T}$ regs mediate their suppressive effect on the immune response both by cell-contact mechanisms that involve specific cell-surface receptors and by the secretion of inhibitory cytokines such as IL-10, TGF- $\beta$ and IL-35 (22). T regs defect was reported in most of autoimmune diseases such as type 1 diabetes, systemic lupus erythematosus, rheumatoid arthritis, multiple sclerosis, myasthenia gravis and chronic immune thrombocytopenia $(23,24)$. Moreover, defect in $\mathrm{T}$ regs has been correlated to higher levels of autoantibodies and immune complexes mediated nephritis in patients with systemic lupus erythematosus $(25,26)$. IE patients suffer from immune system dysregulation evidenced by the production of autoantibodies and immune complexes formation $(8,9)$. Immune complex nephritis is one of the most common and most dangerous complication of infective endocarditis which may result in severe renal injury $(9,18,27)$. The $\mathrm{T}$ regs defect reported herein may explain the presence of theses autoimmune features which IE patients suffer from. The decrease in $\mathrm{T}$ regs in IE patient may underlie some manifestations that complicate the disease course like arthritis, vasculitis and embolization. In agreement with our finding, Araújo et al, reported low production of IL-10 in Staphylococcal IE explaining the exacerbating inflammatory response in this infection (12).

\section{Study limitations:}

Although infective endocarditis is a rare disease, however, the relatively small number of IE patients included in this study is considered a study limitation which did not allow us to find solid correlations between our findings and different disease manifestations. Another limitation is that the study did not include assessment of different cytokines involved in the immune response to IE. Yet, the study sheds the light of the immune system dysfunction in IE that should be further studied on larger cohorts of IE patients.

\section{Conclusion:}

Immune System dysfunction is an important player in IE pathogenesis and contributes to the morbidities reported in this disease. The defect in CD4 memory $\mathrm{T}$ cells may partly explain why some patients pass through this life-threatening disease and their immune system cannot clear the infection. Defect in $\mathrm{T}$ regs present in IE may underlie the autoimmunity reported in those patients. However, extensive studies are still needed to further investigate the role of immune system in IE and correlate the defects in the immune system to the morbidities and mortalities. 
Conflict of interest:

The authors declare that they don't have conflict of interest.

References:

1. Rizk HH, Elamragy AA, Youssef GS, Meshaal MS, Samir A, ElSharkawy A, et al. Clinical features and outcomes of infective endocarditis in Egypt: an 11-year experience at a tertiary care facility. The Egyptian Heart Journal. 2019;71(1):17.

2. Holland TL, Baddour LM, Bayer AS, Hoen B, Miro JM, Fowler VG, Jr. Infective endocarditis. Nat Rev Dis Primers. 2016;2:16059.

3. Meshaal MS, Kassem HH, Samir A, Zakaria A, Baghdady Y, Rizk HH. Impact of routine cerebral CT angiography on treatment decisions in infective endocarditis. PLoS One. 2015;10(3):e0118616.

4. Meshaal MS, Labib D, Said K, Hosny M, Hassan M, Al Aziz SA, et al. Aspergillus endocarditis: Diagnostic criteria and predictors of outcome, A retrospective cohort study. PLoS One. 2018;13(8):e0201459.

5. Abdulhak AAB, Baddour LM, Erwin PJ, Hoen B, Chu VH, Mensah GA, et al. Global and regional burden of infective endocarditis, 1990-2010: a systematic review of the literature. Global Heart. 2014;9(1):131-43.

6. Prendergast BD. The changing face of infective endocarditis. Heart. 2006;92(7):879-85.

7. Osler W. The Gulstonian lectures, on malignant endocarditis. British medical journal. 1885;1(1263):522.

8. Bojalil R, Mazón-González B, Carrillo-Córdova JR, Springall R, Amezcua-Guerra LM. Frequency and clinical significance of a variety of autoantibodies in patients with definite infective endocarditis. JCR: Journal of Clinical Rheumatology. 2012;18(2):67-70.

9. Halpin M, Kozyreva O, Bijol V, Jaber BL. Plasmapheresis for treatment of immune complex-mediated glomerulonephritis in infective endocarditis: a case report and literature review. Clinical nephrology Case studies. 2017;5:26.

10. Ojeda J, López-López L, González A, Vilá LM. Infective endocarditis initially presenting with a dermatomyositis-like syndrome. Case Reports. 2014;2014:bcr2013200865.

11. Mahr A, Batteux F, Tubiana S, Goulvestre C, Wolff M, Papo T, et al. Brief report: prevalence of antineutrophil cytoplasmic antibodies in infective endocarditis. Arthritis \& rheumatology. 2014;66(6):16727 .

12. Araújo IR, Ferrari TCA, Teixeira-Carvalho A, Campi-Azevedo AC, Rodrigues LV, Júnior MHG, et al. Cytokine signature in infective endocarditis. PLoS One. 2015;10(7):e0133631.

13. Layez C, Brunet C, Lépolard C, Ghigo E, Capo C, Raoult D, et al. Foxp3+ CD4+ CD25+ regulatory $\mathrm{T}$ cells are increased in patients with Coxiella burnetii endocarditis. FEMS Immunology \& Medical Microbiology. 2012;64(1):137-9.

14. Habib G, Lancellotti P, Antunes M, Bongiorni M, Casalta J, Del Zotti F, et al. 2015 ESC Guidelines for the management of infective endocarditis. The Task Force for the Management of Infective Endocarditis of the European Society of Cardiology (ESC). Giornale italiano di cardiologia (2006). 2016;17(4):277.

15. Baddour LM, Wilson WR, Bayer AS, Fowler Jr VG, Tleyjeh IM, Rybak MJ, et al. Infective endocarditis in adults: diagnosis, antimicrobial therapy, and management of complications: a scientific statement for healthcare professionals from the American Heart Association. Circulation. 2015;132(15):1435-86.

16. Rubio PEA, Molina RB, Ávila PEA, Mora AG, López CAG. Infective Endocarditis: Inflammatory Response, Genetic Susceptibility, Oxidative Stress, and Multiple Organ Failure. Infective Endocarditis: IntechOpen; 2019.

17. BROWN M, GRIFFIN GE. Immune responses in endocarditis. BMJ Publishing Group Ltd; 1998. 
18. Krishnamurthy S, Chandrasekaran V, Mahadevan S, Priyamvada P, Rajesh NG. Severe acute kidney injury in children owing to infective endocarditis-associated immune complex glomerulonephritis: a report of two cases. Paediatrics and international child health. 2017;37(2):144-7.

19. Megan K.L. MacLeoda ETC, John W. Kapplera,b,c, and Philippa Marracka. CD4 memory T cells: what are they and what can they do? Semin Immunol. 2009;21(2):53-61.

20. MacLeod MK, Kappler JW, Marrack P. Memory CD4 T cells: generation, reactivation and re-assignment. Immunology. 2010;130(1):10-5.

21. Haynes L, Eaton SM, Burns EM, Randall TD, Swain SL. CD4 T cell memory derived from young naive cells functions well into old age, but memory generated from aged naive cells functions poorly. Proceedings of the National Academy of Sciences. 2003;100(25):15053-8.

22. Josefowicz SZ, Lu L-F, Rudensky AY. Regulatory T cells: mechanisms of differentiation and function. Annual review of immunology. 2012;30:531-64.

23. Dominguez-Villar M, Hafler DA. Regulatory $\mathrm{T}$ cells in autoimmune disease. Nature immunology. 2018;19(7):665.

24. Nishimoto T, Satoh T, Takeuchi T, Ikeda Y, Kuwana M. Critical role of CD4+ CD25+ regulatory T cells in preventing murine autoantibody-mediated thrombocytopenia. Experimental hematology. 2012;40(4):27989.

25. Lee JH, Wang LC, Lin YT, Yang YH, Lin DT, Chiang BL. Inverse correlation between CD4+ regulatory T-cell population and autoantibody levels in paediatric patients with systemic lupus erythematosus. Immunology. 2006;117(2):280-6.

26. Tucci M, Stucci S, Strippoli S, Silvestris F. Cytokine overproduction, T-cell activation, and defective T-regulatory functions promote nephritis in systemic lupus erythematosus. BioMed Research International. 2010;2010.

27. Cervi A, Kelly D, Alexopoulou I, Khalidi N. ANCA-associated pauci-immune glomerulonephritis in a patient with bacterial endocarditis: a challenging clinical dilemma. Clinical nephrology Case studies. 2017;5:32.

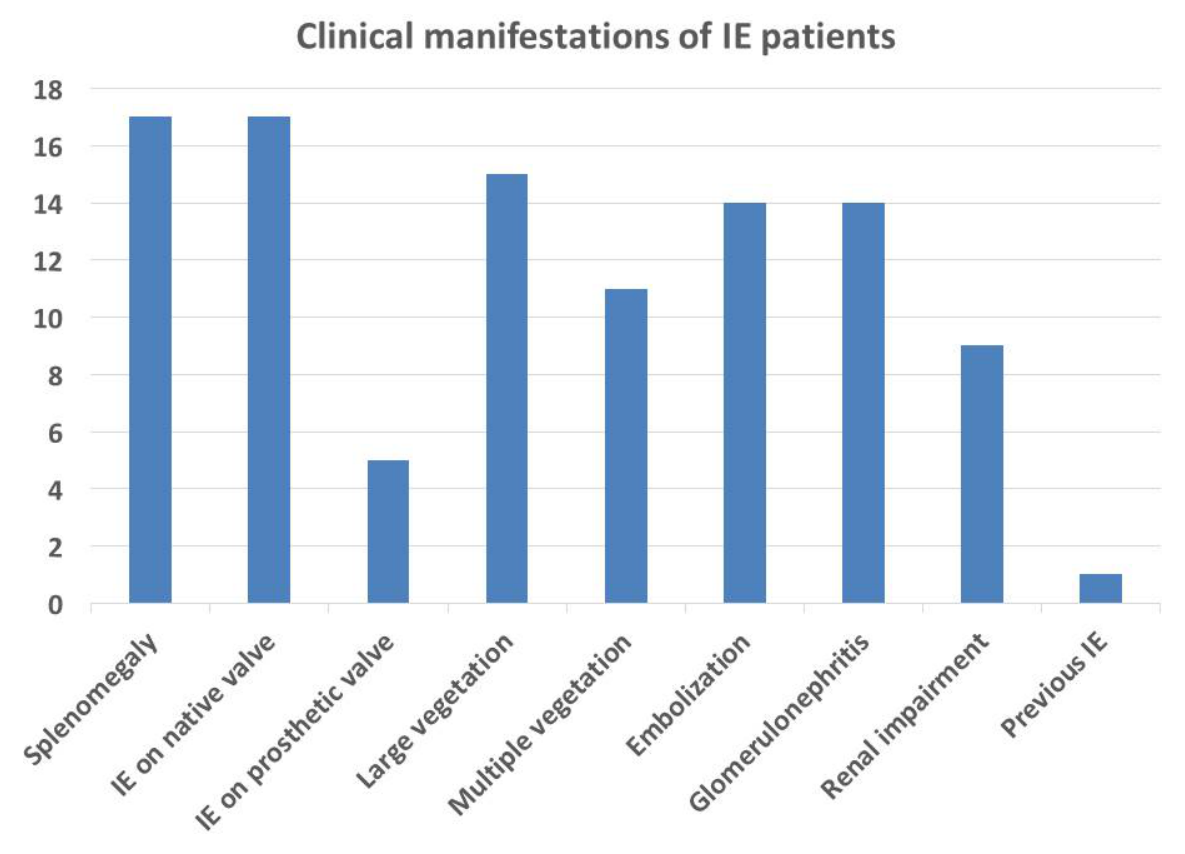


Figure 1: Clinical manifestations among 20 IE patients

\section{Blood culture result in IE patients}

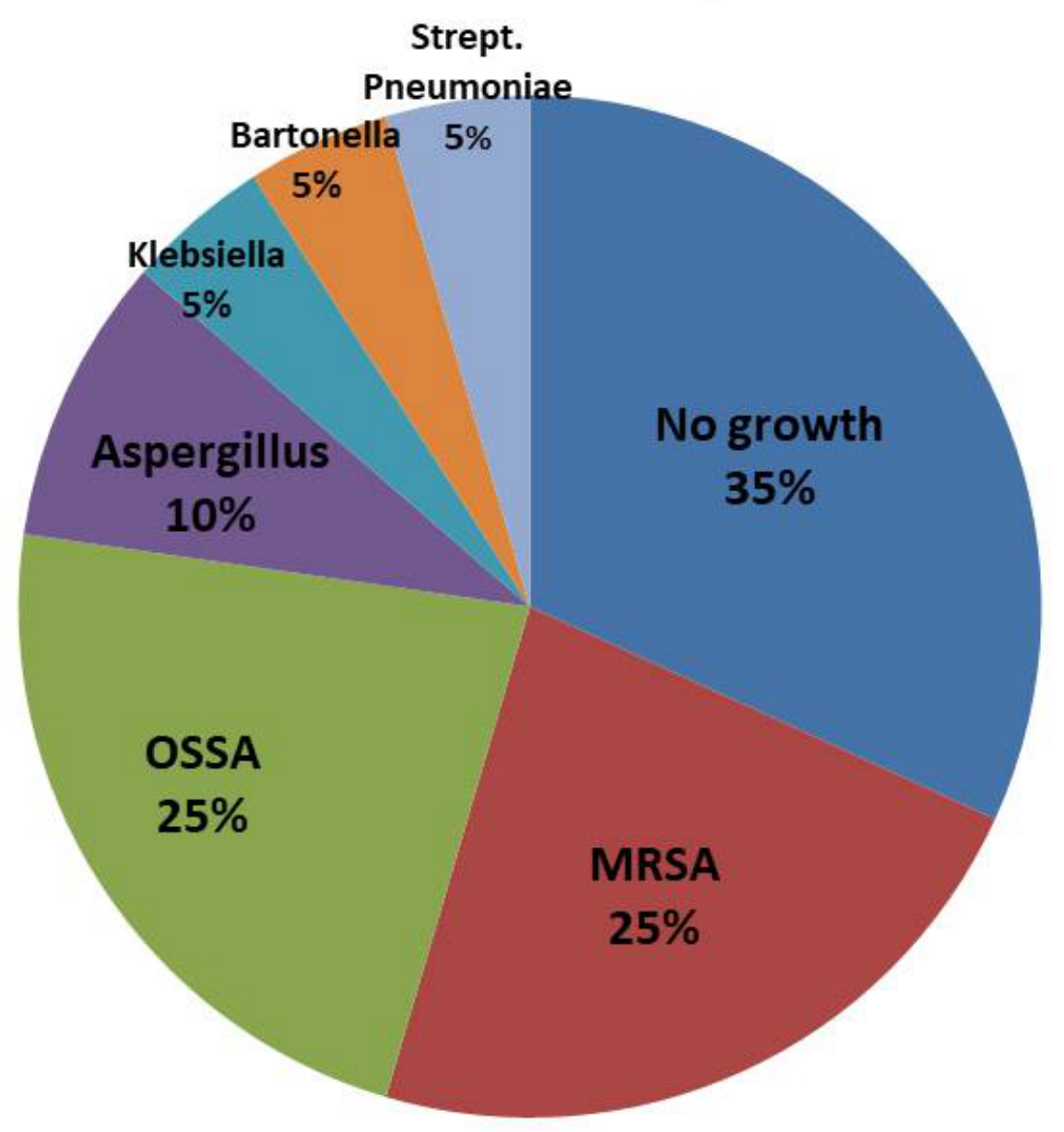

Figure 2: Results of blood culture and detected invading organisms in 20 IE patients 


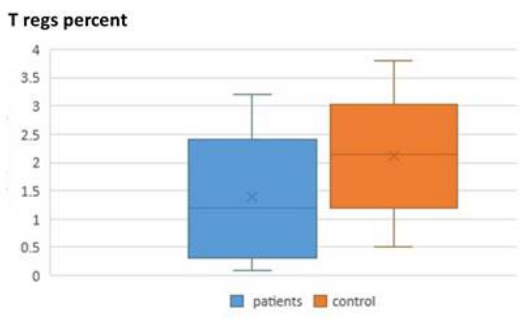

A

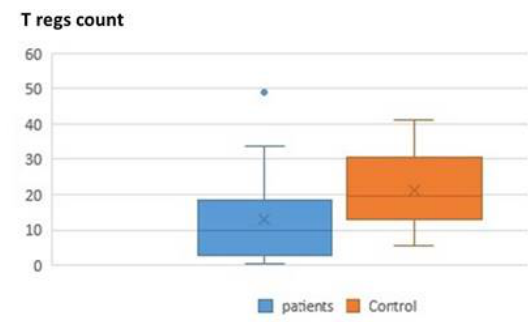

B

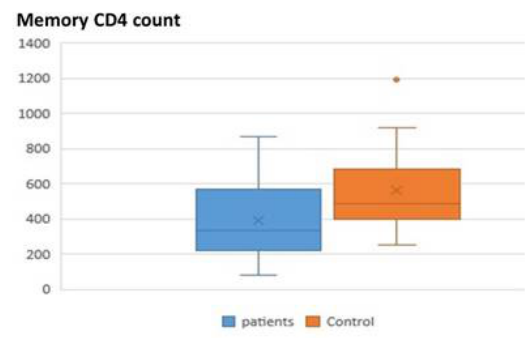

C

Figure 2: Comparison between IE patients and healthy control showing the interquartile range, median and mean regarding (A) Tregs percentage, (B) Tregs count and (C) memory CD45ROCD4+ T cells count

\section{Clinical manifestations of IE patients}

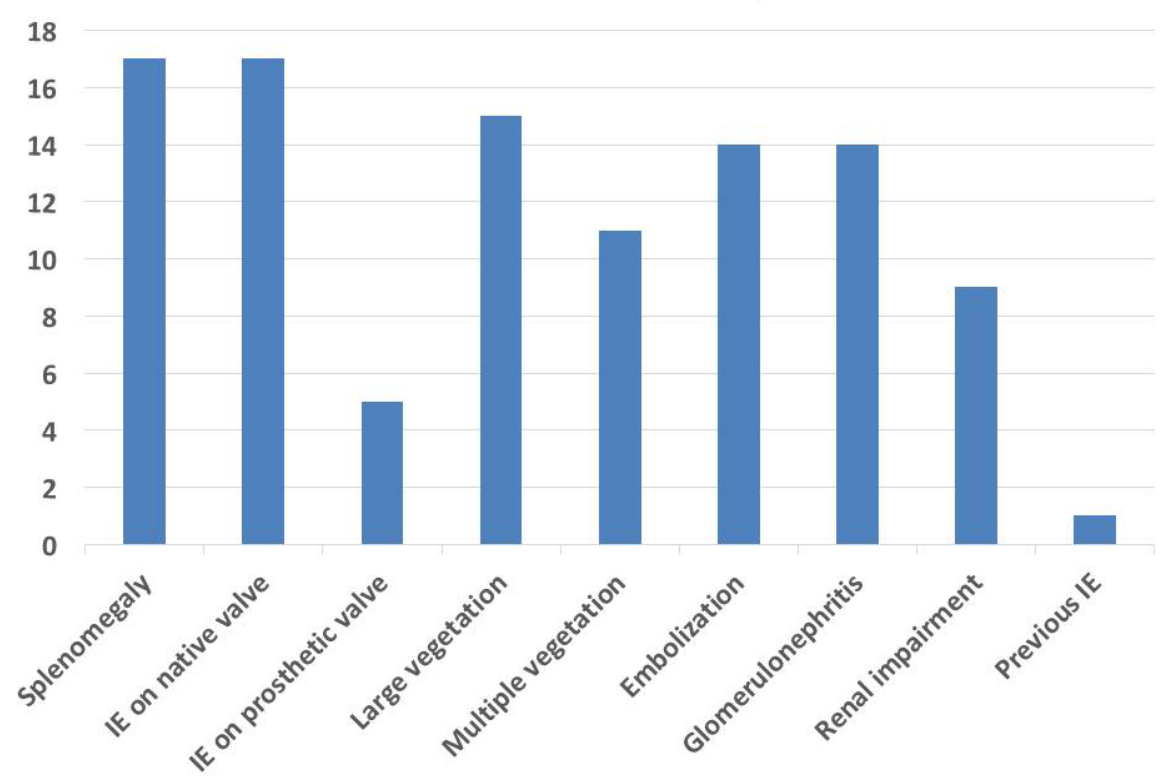




\section{Blood culture result in IE patients}

Strept.

Pneumoniae

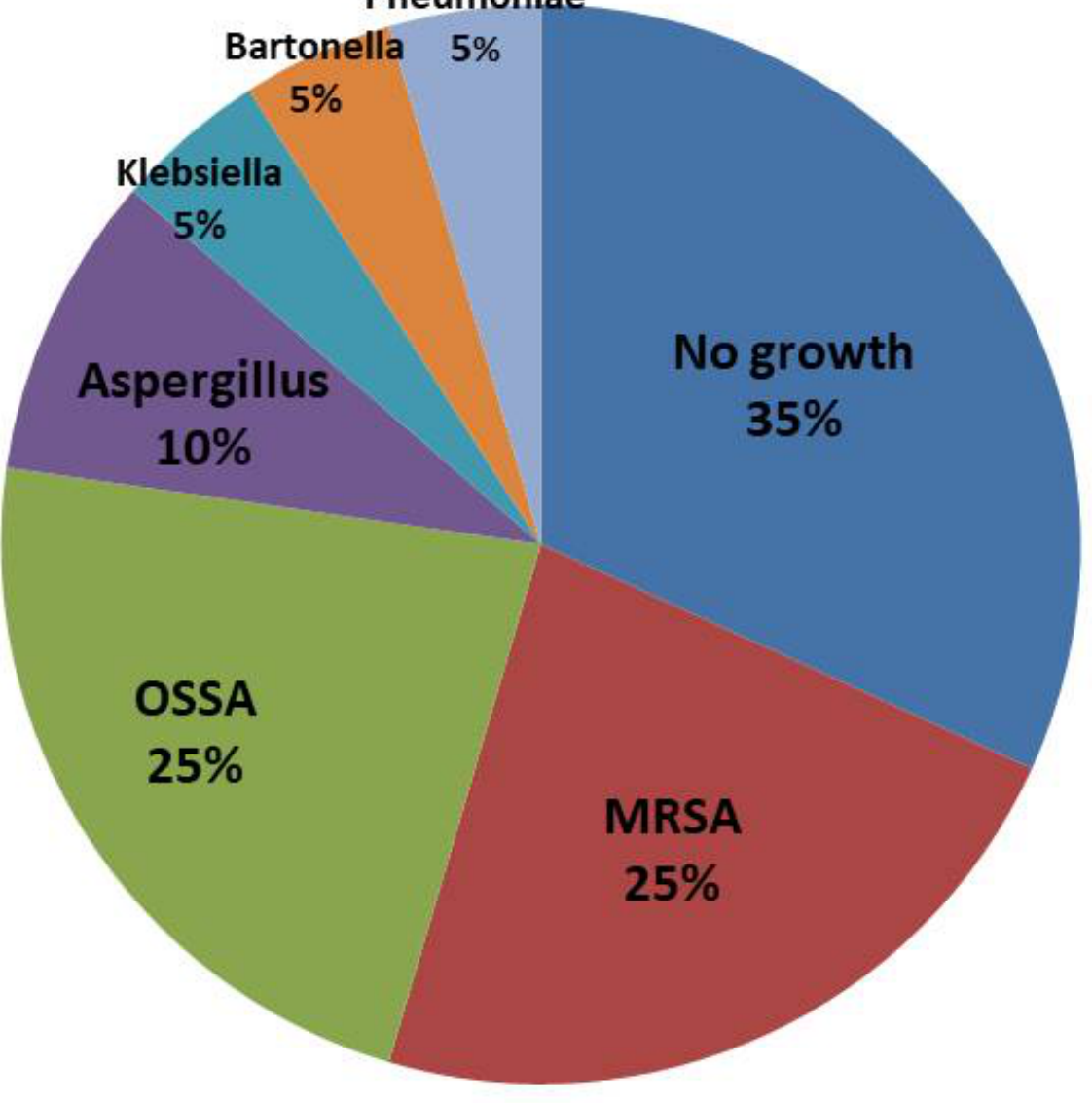




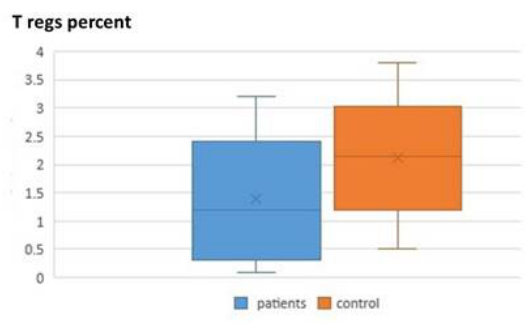

A

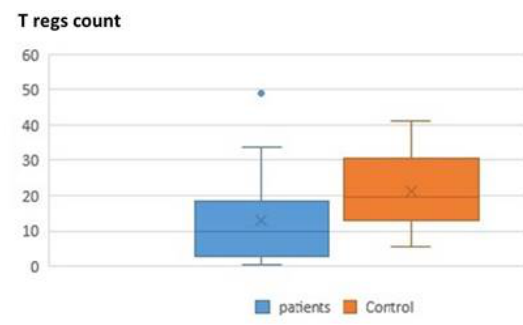

B

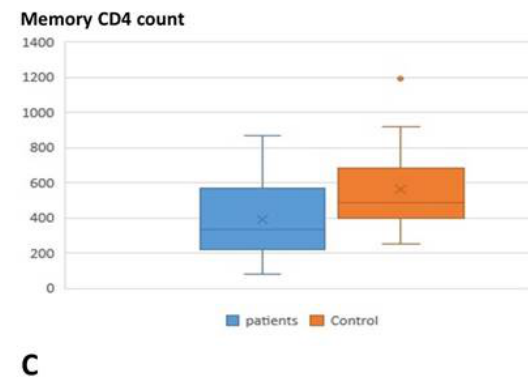

